\title{
Dancing is allowed in steady-states
}

\author{
Non-equilibrium systems have steady-state distributions and non-steady \\ dynamics
}

Authors: Tanniemola B. Liverpool

arXiv:1810.10980

\begin{abstract}
Recommended with a Commentary by Ramin Golestanian, Max
Planck Institute for Dynamics and Self-Organization

(Göttingen) and University of Oxford
\end{abstract}

Is it possible to describe, characterize, and understand non-equilibrium systems the way we do it for equilibrium systems? In general, this is a formidable task, but when it is possible how would we do it? In a recent preprint, Liverpool argues that a viewpoint that focuses on "typical" trajectories of a complex system might help us shed some light on the characteristic behaviour of its steady-state.

Consider a system described by the following Fokker-Planck equation

$$
\partial_{t} \mathcal{P}(\boldsymbol{x}, t)+\nabla \cdot[\boldsymbol{v}(\boldsymbol{x}, t) \mathcal{P}-D \nabla \mathcal{P}]=0
$$

which is a statement of conservation of probability. This conservation law can be rewritten as

$$
\partial_{t} \mathcal{P}(\boldsymbol{x}, t)+\nabla \cdot\left[\boldsymbol{v}_{\text {eff }}(\boldsymbol{x}, t) \mathcal{P}\right]=0,
$$

in terms of the effective drift velocity

$$
\boldsymbol{v}_{\text {eff }}(\boldsymbol{x}, t)=\boldsymbol{v}(\boldsymbol{x}, t)-D \nabla \ln \mathcal{P}(\boldsymbol{x}, t)
$$

that represents both the deterministic and the stochastic contributions to the velocity. When the drift velocity does not have explicit time dependence, the system might reach a steadystate. To look for it, we can set $\partial_{t} \mathcal{P}_{\mathrm{ss}}=0$ and obtain the solution for the probability distribution in steady-state through $\nabla \cdot\left(\boldsymbol{v}_{\text {eff }} \mathcal{P}_{\text {ss }}\right)=0$, which yields a time-independent distribution $\mathcal{P}_{\mathrm{ss}}(\boldsymbol{x})$.

While the distribution does not appear to have any dynamical information encoded in it - since the quantities of interest are time-independent - the steady-state in general might exhibit complex dynamics. This is often the case in active matter, such as active nematic systems that exhibit a rich dynamical steady-state in which $+1 / 2$ and $-1 / 2$ defects are constantly created and annihilated $[1,2,3]$.

What Liverpool proposes is that by examining the following dynamical system

$$
\frac{\mathrm{d} \boldsymbol{r}}{\mathrm{d} t}=\boldsymbol{v}_{\mathrm{eff}}(\boldsymbol{r}),
$$


one is able to gain insight into what typical dynamical trajectories might look like if one were to monitor the real-time dynamics of the steady-state, say in a simulation or in an experiment. It is important to note that this dynamical system includes in its formulation explicit information about the steady-state distribution $\mathcal{P}_{\mathrm{ss}}$, through $\boldsymbol{v}_{\text {eff }}(\boldsymbol{r})=\boldsymbol{v}(\boldsymbol{r})-D \nabla \ln \mathcal{P}_{\mathrm{ss}}(\boldsymbol{r})$.

To demonstrate the strength of this method, one can take a step back and show how one can tease out the relevant dynamical information about a system from the above dynamical system, using existing exact solutions for $\mathcal{P}(\boldsymbol{x}, t)$. For a free Brownian particle, we have

$$
\frac{\mathrm{d} \boldsymbol{r}}{\mathrm{d} t}=\frac{\boldsymbol{r}}{2 t}
$$

which yields $|\boldsymbol{r}|=(6 D t)^{1 / 2}$ as expected. For a particle in a harmonic potential $U(\boldsymbol{x})=k \boldsymbol{x}^{2} / 2$, we have $\boldsymbol{v}(\boldsymbol{x})=-\nabla U / \zeta=-k \boldsymbol{x} / \zeta$, where $\zeta$ is the friction coefficient, and related to the diffusion coefficient $D$ through the Einstein relation $D=k_{\mathrm{B}} T / \zeta$. We can construct the dynamical system as

$$
\frac{\mathrm{d} \boldsymbol{r}}{\mathrm{d} t}=-\frac{k \boldsymbol{r}}{\zeta}+\frac{k \boldsymbol{r}}{\zeta\left[1-e^{-2 k t / \zeta}\right]}
$$

and solve it to obtain $|\boldsymbol{r}|=\sqrt{3 \frac{k_{\mathrm{B}} T}{k}\left[1-e^{-2 k t / \zeta}\right]}$, which gives the appropriate short-time and long-time asymptotic limits.

In the above examples, time-independent $\boldsymbol{v}(\boldsymbol{r})$ gave rise to time-dependent trajectories $\boldsymbol{r}(t)$ because we essentially studied approach to steady-state by using information about the time-dependence of the distributions. One can imagine, however, that already in the steady-state more complicated dynamics might emerge, such as oscillations, as Liverpool demonstrate with the example of a noisy Brusselator. This method relies on the availability of $\ln \mathcal{P}_{\mathrm{ss}}$, which Liverpool argues, can be calculated perturbatively in the spirit of a Landau expansion.

It is possible to generalize the description to an infinite dimensional Hilbert space, e.g. representing stochastic dynamics of a field in a continuum. Liverpool chooses the dynamics of active nematics in the steady-state and shows that by adapting this formalism one can identify patterns of dynamic activity in the steady-state that resemble the dynamic behaviour of active nematics as observed in the simulations and experiments $[1,2,3]$. He shows that by going beyond the linear stability analysis one can identify the following two effects of the activity on the nematic field: it excites some of the soft (Goldstone) modes of the brokensymmetry phase and then generates nearly periodic dynamics of these modes. Since the periodicity of each mode is different, this leads to formation of complex dynamical patterns.

The specific examples in this work suggest that the method can have the potential to help us uncover the underlying dynamical properties of many complex active matter systems in steady-state, and perhaps beyond that upon suitable generalizations. This is a very promising prospect at the time when active matter research is unraveling ever so vigorously.

\section{References}

[1] T. Sanchez, D. T. N. Chen, S. J. De Camp, M. Heymann, and Z. Dogic, Nature (London) 491, 431 (2012). 
[2] L. Giomi, M. J. Bowick, X. Ma, and M. C. Marchetti, Phys. Rev. Lett. 110, 228101 (2013).

[3] S. P. Thampi, R. Golestanian, and J. M. Yeomans, Phys. Rev. Lett. 111, 118101 (2013). 